\title{
The Mobility of a Hydrogen Atom on an Icy Mantle
}

\author{
Koichi Masuda
}

The Graduate School of Sci. and Technol., Kobe Univ., Nada 657, Japan

\begin{abstract}
It is probable that most molecules in space are formed on the surface of small solid grains in dense molecular clouds. Such grains have generally accumulated icy mantles inside dark clouds. We have investigated the dynamical behavior of a hydrogen atom impinging on the mantle consisting of amorphous water ice based on an MD (Molecular Dynamics) computer simulation to estimate the structure of the resulting grains with the icy mantles. We have found that the hydrogen atoms impinging on the surface of amorphous water ice were easily trapped in a dent of the surface and, consequently they are fixed there firmly. Our results, which neglect tunneling, suggest that the migration of hydrogen atoms over a large region of the surface of icy grains may be less common than is often assumed.
\end{abstract}

\section{Introduction}

In space, it is likely that most molecules are formed on the surface of small solid grains. Such grains generally have icy mantles which accumulate on the seeds of solid particles inside dark clouds. It is believed that $\mathrm{H}_{2} \mathrm{O}$-ice itself is formed by hydrogenaticn of incident oxygen atoms. From both laboratory studies and astronomical observations, it is also apparent that the chemical reactions to produce heavier molecules occur between the species trapped in or on the icy surface. Most of the astronomical observations tell us that the accumulated icy dust mantles are expected to be amorphous ice rather than crystalline, because of the low temperature of the environment (e.g. Leger et al. 1979).

Leitch-Devlin and Williams (1985) studied the sticking coefficients, and furthermore the mobilities of additional atoms $\left(\mathrm{H}, \mathrm{H}_{2}, \mathrm{C}\right.$, etc.) at the surfaces of crystalline solids (graphite, silicate, oxide, etc.) with full quantum mechanical treatment (Leitch-Devlin and Williams 1984). Buch and Zhang (1991) simulated the sticking probability of $\mathrm{H}$ and $\mathrm{D}$ atoms on amorphous water clusters containing $115 \mathrm{H}_{2} \mathrm{O}$ molecules.

The real dust grains, however, have amorphous structure and are much larger than clusters containing several hundred molecules. In this study, we investigated the dynamical behaviors of a hydrogen atom impinging on an amorphous water ice mantle. The dust mantle is assumed to have a slab shape consisting of amorphous water ice. We produce the amorphous water slab modeled by a MD (Molecular Dynamics) computer simulation for a many body system. After making the slab, a hydrogen atom is thrown on the surface of the slab to study its dynamical behavior. 


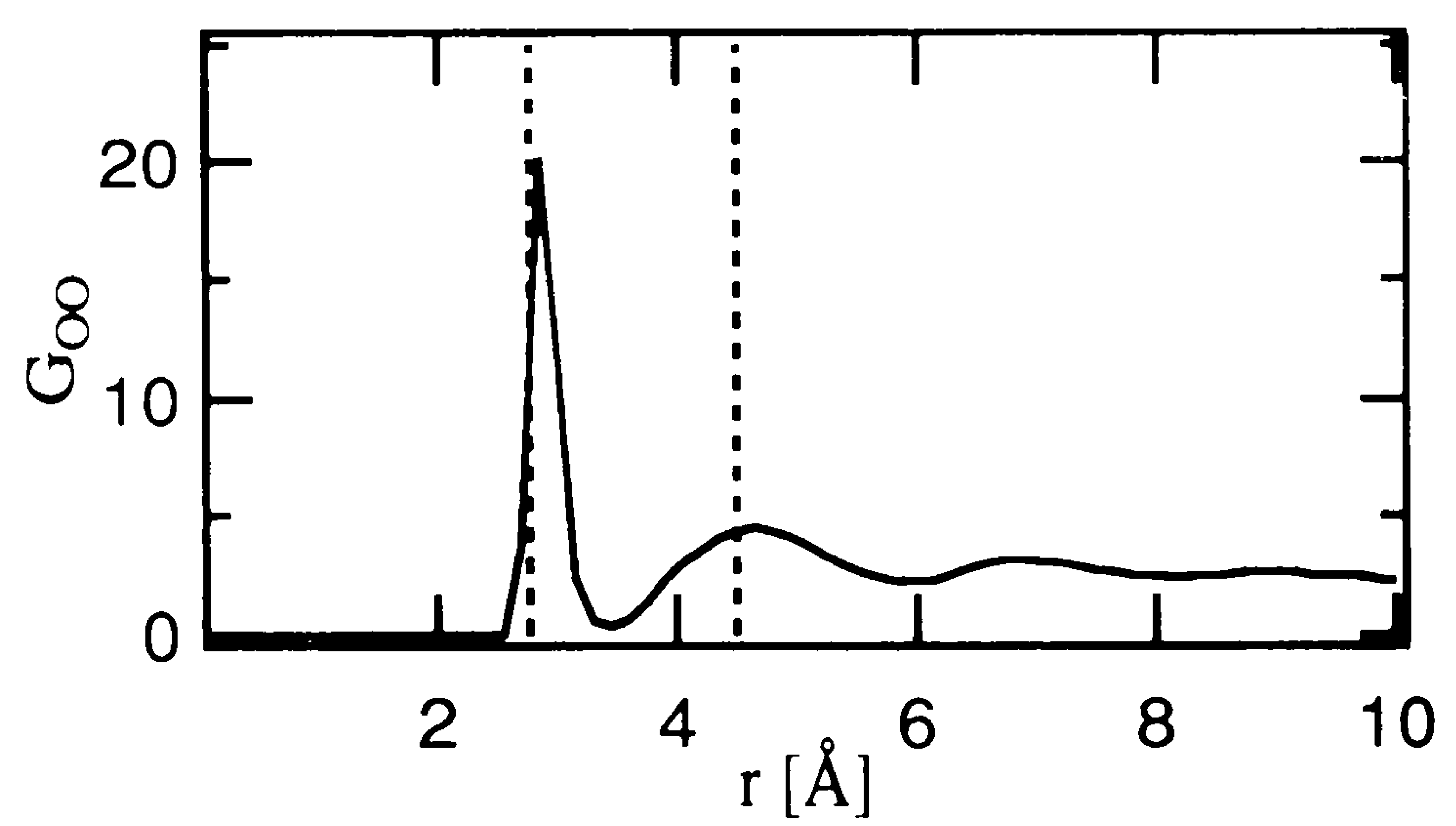

Figure 1. Radial distribution function of oxygen atoms $\mathrm{G}_{\mathrm{OO}}$ in the amorphous water slab made by our simulation (solid curve). Two vertical dotted lines indicate the positions of the peaks of oxygen concentration found from $\mathrm{x}$-ray diffraction experiments (Narten et al. 1975)

\section{The amorphous water ice slab}

As the inter-molecules potential model of water, we employ the MCY (Matsuoka, Clementi and Yoshimine) potential model (Matsuoka et al. 1976). This potential model is known to yield reasonable energetic and structural features measured empirically for dimers, and yield the complexes in the gas phase, referring to the experimental data and the outcomes of high-quality ab initio quantum-mechanical calculations.

Since the mantle of dust grains modeled by the amorphous water ice slab should have infinite surface area, we carry out the simulation producing the slab under the periodic boundary conditions. The basic slab has about $40 \AA \times 40 \AA$ $\times 20 \AA$ volume and consists of 1,000 water molecules. The temperature of this slab is set to $70 \mathrm{~K}$. It is known that there are two types of amorphous water ice having different structure (e.g. Jenniskens et al 1995). Below $35 \mathrm{~K}$, it has high density structure (about $1.1 \mathrm{~g} / \mathrm{cm}^{3}$ ). But over $35 \mathrm{~K}$, like this simulation, it has low density (about $0.9 \mathrm{~g} / \mathrm{cm}^{3}$ ). Although inside the typical dark clouds the temperature is low (about $10 \mathrm{~K}$ ). we employ $70 \mathrm{~K}$ because we cannot take into account low temperature quantum effects and the simulation at such high temperature takes less time for computation.

The resulting amorphous water slab has low density $\left(0.94 \mathrm{~g} / \mathrm{cm}^{3}\right)$. Furthermore, we have found that the water mantle shows amorphous structure and hard roughiness (cf. Fig.2.). The radial distribution function of oxygen atoms $G_{O O}$ in this amorphous water slab is shown in Fig.1. In this figure, two vertical dotted lines indicate the positions of the peaks of the radial distribution function of oxygen atoms found from x-ray diffraction experiments for bulk amorphous water ice (Narten et al. 1975). Since there are good agreements of the peaks between the result of our slab and the experiment, we can use our slab as a model for a real amorphous water ice slab. 

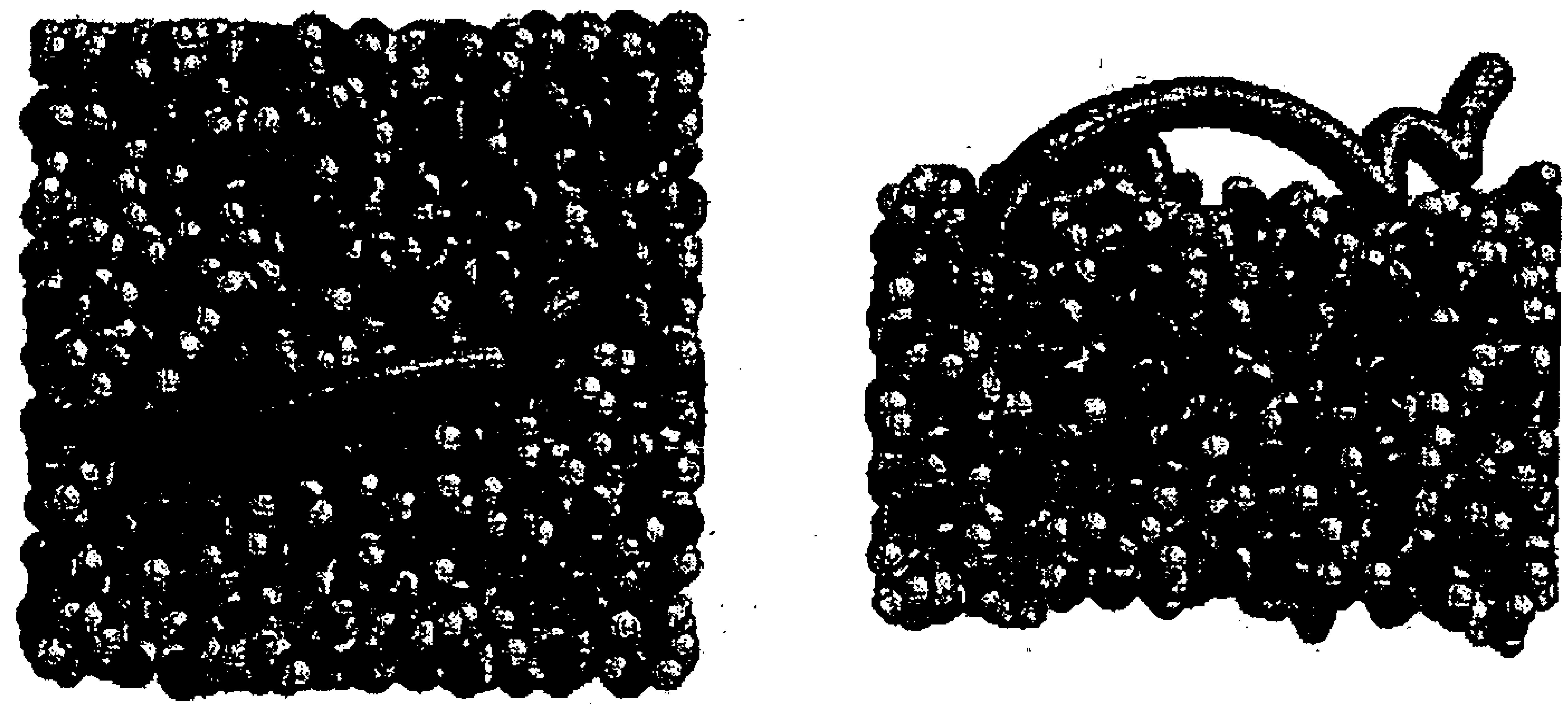

Figure 2. Two views of the trajectory of the impinging hydrogen atom. The dark spheres are oxygen atoms, and light spheres are hydrogen. The silver tube indicates the trajectory of the hydrogen atom. The hydrogen atom is trapped in one of potential dents after several bounces on the surface.

\section{3. 'Impinging a hydrogen atom}

Using the above ainorphous water slab, we investigate the surface properties of the dust mantle for an impinging hydrogen atom. In order to simúlate the behavivior of the hydrogen atom against the ensemble of the water molecules; we employ the potential model between a hydrogen atom and a water molecule modeled by Zhang et al. (1991). This potential model was also made by the $a b$ initio calculations. An impinging hydrogen atom has thermal velocity of 70K. A lack of enough computer capacity prevented us from using the Maxwell distribution of velocities, so a constant impinging velocity was applied. The incident angle of the impinging hydrogen atom; was selected randomly. The simulation has been carried out under the periodic boundary condition.

\section{Results and discussions}

We have done the simulation for the dynamical behavior of impinging hydrogen atoms on the amorphous water slab four times with changing its, incident direction. In these simulations, scattering and re-ejection of the impinging hydrogen atom did not occur. "Fig.2 shows the snapshots of the one of these simulations. We have found from the trajectory of the hydrogen atom in Fig.2 that the impinging hydrogen atom adheres to one place on the surface after several bounces on it. The potential field which is felt by the impinging hydrogen atom due'to the amorphous slab is' shown in Fig.3. The water molecules exist in the black region. The depth of the potential is indicated by a darkness of the gradation region, where deeper is darker. The white dotted rectangle indicates the basic cell for simulation. The trajectory of test hydrogen shown in Fig.2 is 


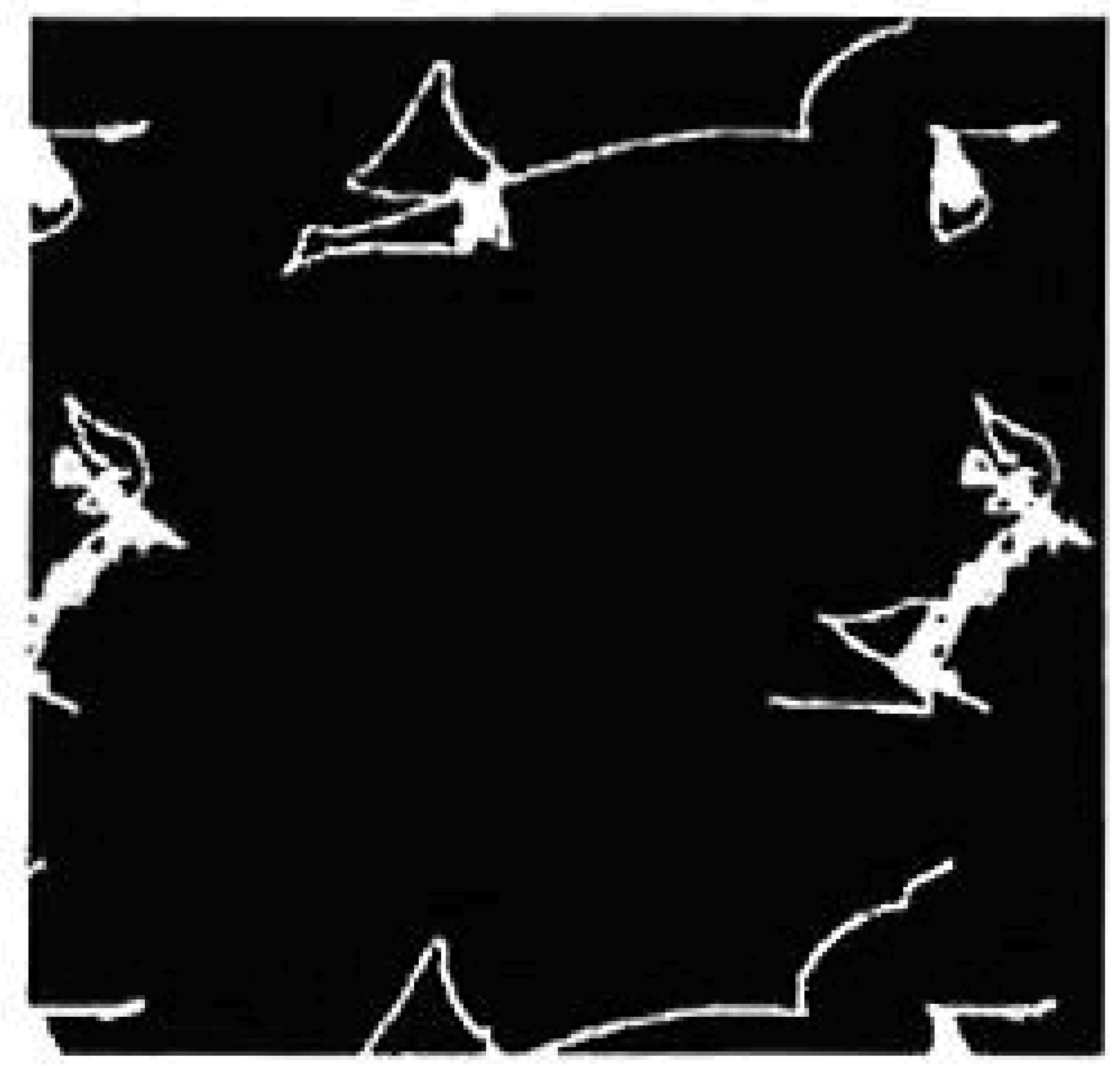

Figure 3. The potential field and trajectories of the impinging hydrogen atoms due to the amorphous slab at the arbitrary height. Black regions mean that there are water molecules. Gradation regions mean the depth of the potential which is deeper at the darker place. The trajectories of the impinging hydrogen atom are given by white curves. White dotted rectangle indicates the basic cell for this simulation.

illustrated by the white curve in Fig. 3. Similar curves in the upper and lower parts occur due to our periodic boundary conditions. All these simulations indicate that the impinging hydrogen atom is trapped in one of the potential dents after bounding several times on the surface of the amorphous slab. This evidence may suggest that the diffusion of the hydrogen atoms over a large surface region seems to be difficult. Moreover, it is expected that the chemical reactions producing the hydrogenated molecules preferentially occur inside such dents.

In this study, we did not take into account quantum effects, although it is well known that the tunneling effect is relatively important for hydrogen atom. Moreover, inside dark clouds, a typical temperature is lower than that used here, i.e. about $10 \mathrm{~K}$. We will examine the quantum effect at such low temperature in future work.

Acknowledgments. I would like to thank Atto Laaksonen for his code in the CCP5 program library in the computer center of Kyoto University, and Tadashi Mukai and Mayo Greenberg for their discussion and comments.

\section{References}

Buch, V., \& Zhang, Q. 1991, $A p j, 379,647$

Jenniskens, P., Blake, D. F., Wilson, M. A., \& Pohorille, A. 1995, Apj (accepted) Leger, A., Klein, J., Cheveigne, S. de, Guinet, C., Defourneau, D., \& Belin, M. $1979, A \& A, \mathbf{7 9}, 256$

Leitch-Devlin, M. A., \& Williams, D. A. 1985, MNRAS, 213, 295

Matsuoka, O., Clementi, E., \& Yoshimine M. 1976, J.Chem.Phys., 64, 1351

Narten, A. H., C. G. Venkatesh, C. G., \& Rice, S. A. 1975, J.Chem.Phys., 64, 1106

Zhang, Q., Sabelli, N., \& Buch, V. 1991, J.Chem.Phys., 95, 1080 